\title{
Excited States of the Nucleon in 2+1 Flavor QCD
}

\author{
M. S. Mahbub ${ }^{a b}$, W. Kamleh ${ }^{a}$, D. B. Leinweber ${ }^{*}$, P. J. Moran ${ }^{a}$, A. G. Williams ${ }^{a}$ \\ a Special Research Centre for the Subatomic Structure of Matter, Adelaide, South Australia 5005, \\ Australia, and Department of Physics, University of Adelaide, South Australia 5005, Australia. \\ ${ }^{b}$ Department of Physics, Rajshahi University, Rajshahi 6205, Bangladesh. \\ E-mail: md.mahbubeadelaide.edu.au, \\ waseem.kamleh@adelaide.edu.au, \\ derek.leinweber@adelaide.edu.au, \\ peter.moranealumni.adelaide.edu.au, \\ anthony.williamseadelaide.edu.au
}

Recent developments on the determination of the spin-1/2 spectrum of the nucleon in full QCD are presented. Our focus is on the PACS-CS $2+1$ flavor configurations made available through the ILDG. Using correlation matrix techniques, in which a wide variety of gauge-invariant Gaussiansmeared fermion-propagator sources and sinks are considered, excited states are determined. We consider several correlation matrices of various sizes, each constructed with a different set of basis interpolators, in order to demonstrate the invariance of the eigenstates on the basis choice. Of particular interest is the approach to the elusive Roper resonance and we report preliminary results in full QCD.

The XXVIII International Symposium on Lattice Field Theory, Lattice2010

June 14-19, 2010

Villasimius, Italy

\footnotetext{
* Speaker.
} 


\section{Introduction}

The first positive-parity excited state of the nucleon, known as the Roper resonance, $N \frac{1}{2}^{+}(1440$ $\mathrm{MeV}$ ) $\mathrm{P}_{11}$, has presented a long-standing puzzle since its discovery in the 1960 's due to its lower mass compared to the adjacent negative parity, $N \frac{1}{2}^{-}(1535 \mathrm{MeV}) \mathrm{S}_{11}$, state. In constituent quark models with harmonic oscillator potentials, the lowest-lying odd-parity state naturally occurs below the $\mathrm{P}_{11}$ state $[1,2]$. In nature the Roper resonance is almost $100 \mathrm{MeV}$ below the $\mathrm{S}_{11}$ state.

Lattice QCD is very successful in computing many properties of hadrons from first principles. In particular, the ground state of the hadron spectrum is a well understood problem. However, the excited states still provide a significant challenge. The first analysis of the positive parity excitation of the nucleon was performed in Ref. [3] using Wilson fermions and an operator product expansion spectral ansatz. Since then several attempts have been made to address these issues in the lattice framework.

The "variational method" $[4,5]$ is one of the state-of-the-art approaches to hadron spectroscopy, which is based on a correlation matrix analysis. The identification of the Roper state with this method wasn't successful until recently. In Refs. [6, 7] a low-lying Roper state has been identified with this approach by employing a diverse range of smeared-smeared correlation functions. Also, with this method, a physical level ordering between the Roper and $N \frac{1}{2}^{-}$ground state is observed in Ref. [8].

Recent developments of algorithms and computational power have enabled us to explore this physics in full QCD. Some recent full QCD results has been presented in Refs. [9, 10, 11]. In this paper, we present preliminary results for the excited states of the nucleon using the PACS-CS $2+1$ flavor configurations [12]. The results are presented from the variational method using various fermion source and sink smearings to construct correlation matrices.

\section{Variational Method}

The two point correlation function matrix for a $\vec{p}=0$ baryon can be written as

$$
\begin{aligned}
G_{i j}^{ \pm}(t) & =\sum_{\vec{x}} \operatorname{Tr}_{\mathrm{sp}}\left\{\Gamma_{ \pm}\left\langle\Omega\left|\chi_{i}(x) \bar{\chi}_{j}(0)\right| \Omega\right\rangle\right\} \\
& =\sum_{\alpha} \lambda_{i}^{\alpha} \bar{\lambda}_{j}^{\alpha} e^{-m_{\alpha} t}
\end{aligned}
$$

where Dirac indices are implicit. Here, $\lambda_{i}^{\alpha}$ and $\bar{\lambda}_{j}^{\alpha}$ are the couplings of interpolators $\chi_{i}$ and $\bar{\chi}_{j}$ at the sink and source respectively and $\alpha$ enumerates the energy eigenstates with mass $m_{\alpha} . \Gamma_{ \pm}=$ $\frac{1}{2}\left(\gamma_{0} \pm 1\right)$ projects the parity of the eigenstates.

Since the only $t$ dependence comes from the exponential term, one can seek a linear superposition of interpolators, $\bar{\chi}_{j} u_{j}^{\alpha}$, such that,

$$
G_{i j}\left(t_{0}+\triangle t\right) u_{j}^{\alpha}=e^{-m_{\alpha} \triangle t} G_{i j}\left(t_{0}\right) u_{j}^{\alpha},
$$

for sufficiently large $t_{0}$ and $t_{0}+\triangle t$. More detail can be found in Refs. [13, 14, 15]. Multiplying the above equation by $\left[G_{i j}\left(t_{0}\right)\right]^{-1}$ from the left leads to an eigenvalue equation,

$$
\left[\left(G\left(t_{0}\right)\right)^{-1} G\left(t_{0}+\triangle t\right)\right]_{i j} u_{j}^{\alpha}=c^{\alpha} u_{i}^{\alpha},
$$


where $c^{\alpha}=e^{-m_{\alpha} \triangle t}$ is the eigenvalue. Similar to Eq. (2.4), one can also solve the left eigenvalue equation to recover the $v^{\alpha}$ eigenvector,

$$
v_{i}^{\alpha}\left[G\left(t_{0}+\triangle t\right)\left(G\left(t_{0}\right)\right)^{-1}\right]_{i j}=c^{\alpha} v_{j}^{\alpha} .
$$

The vectors $u_{j}^{\alpha}$ and $v_{i}^{\alpha}$ diagonalize the correlation matrix at time $t_{0}$ and $t_{0}+\triangle t$ making the projected correlation matrix,

$$
v_{i}^{\alpha} G_{i j}^{ \pm}(t) u_{j}^{\beta} \propto \delta^{\alpha \beta} .
$$

The parity projected, eigenstate projected correlator,

$$
G_{ \pm}^{\alpha} \equiv v_{i}^{\alpha} G_{i j}^{ \pm}(t) u_{j}^{\alpha},
$$

is then analyzed using standard techniques to obtain the masses of the states, $\alpha$.

\section{Simulation Details}

The PACS-CS $2+1$ flavor dynamical-fermion configurations [12] are used. The non-perturbatively $\mathscr{O}(a)$-improved Wilson fermion action and Iwasaki-gauge action [16] are employed. The lattice volume is $32^{3} \times 64$, with $\beta=1.90$ providing lattice spacing $a=0.0907 \mathrm{fm}$ and $c_{S W}=1.715$ [17]. Five kappa values with degenerate up down quarks have been considered, i.e. $k_{u d}=0.13700$, $0.13727,0.13754,0.13770,0.13781$, with $k_{s}=0.13640$. In this paper, we also consider the Sommer scale [18], with $a$ as described in Ref. [12]. The results are presented for $k_{u d}=0.13754,0.13770$, for which ensembles of 350 configurations are considered. As with the quenched case [6], various levels of gauge invariant Gaussian smearing [19] are applied at the fermion source $(t=16)$ and at the sink. We consider 4, 9, 16, 25, 35, 50, 70, 100, 125, 200, 400, 800, 1600 sweeps, corresponding to rms radii in lattice units of 1.20, 1.79, 2.37, 2.96, 3.50, 4.19, 4.95, 5.920, 6.63, 8.55, 12.67, 15.47, 16.00. The error analysis is performed using the jackknife method, where the $\chi^{2} /$ dof is obtained via a covariance matrix analysis. Our fitting method is discussed extensively in Refs. [7, 14].

The nucleon interpolator we consider here is the local scalar-diquark interpolator [3, 20],

$$
\chi_{1}(x)=\varepsilon^{a b c}\left(u^{T a}(x) C \gamma_{5} d^{b}(x)\right) u^{c}(x) .
$$

\section{Results}

We consider several $4 \times 4$ correlation matrices. Each matrix is constructed with different sets of correlation functions, each set element corresponding to a different number of sweeps of gauge invariant Gaussian smearing at the source and sink of the $\chi_{1} \bar{\chi}_{1}$ correlators. This provides a large basis of operators as described in Table 1, providing a wide range of overlap with energy eigenstates.

Consistency of the extracted masses is manifest in Fig. 1. In particular, the ground and Roper states are robust. The highest energy state shows some basis dependency (smearing dependency) which is to be expected as this state must accommodate all remaining spectral strength [6]. 
Table 1: $4 \times 4$ correlation matrix bases.

\begin{tabular}{c|cccccccccc}
\hline Sweeps $\rightarrow$ & 16 & 25 & 35 & 50 & 70 & 100 & 125 & 200 & 400 & 800 \\
\hline Basis No. $\downarrow$ & \multicolumn{10}{|c}{ Bases } \\
\hline 1 & 16 & - & 35 & - & 70 & 100 & - & - & - & - \\
2 & 16 & - & 35 & - & 70 & - & 125 & - & - & - \\
3 & 16 & - & 35 & - & - & 100 & - & 200 & - & - \\
4 & 16 & - & 35 & - & - & 100 & - & - & 400 & - \\
5 & 16 & - & - & 50 & - & 100 & 125 & - & - & - \\
6 & 16 & - & - & 50 & - & 100 & - & 200 & - & - \\
7 & 16 & - & - & 50 & - & - & 125 & - & - & 800 \\
8 & - & 25 & - & 50 & - & 100 & - & 200 & - & - \\
9 & - & 25 & - & 50 & - & 100 & - & - & 400 & - \\
10 & - & - & 35 & - & 70 & - & 125 & - & 400 & - \\
\hline
\end{tabular}

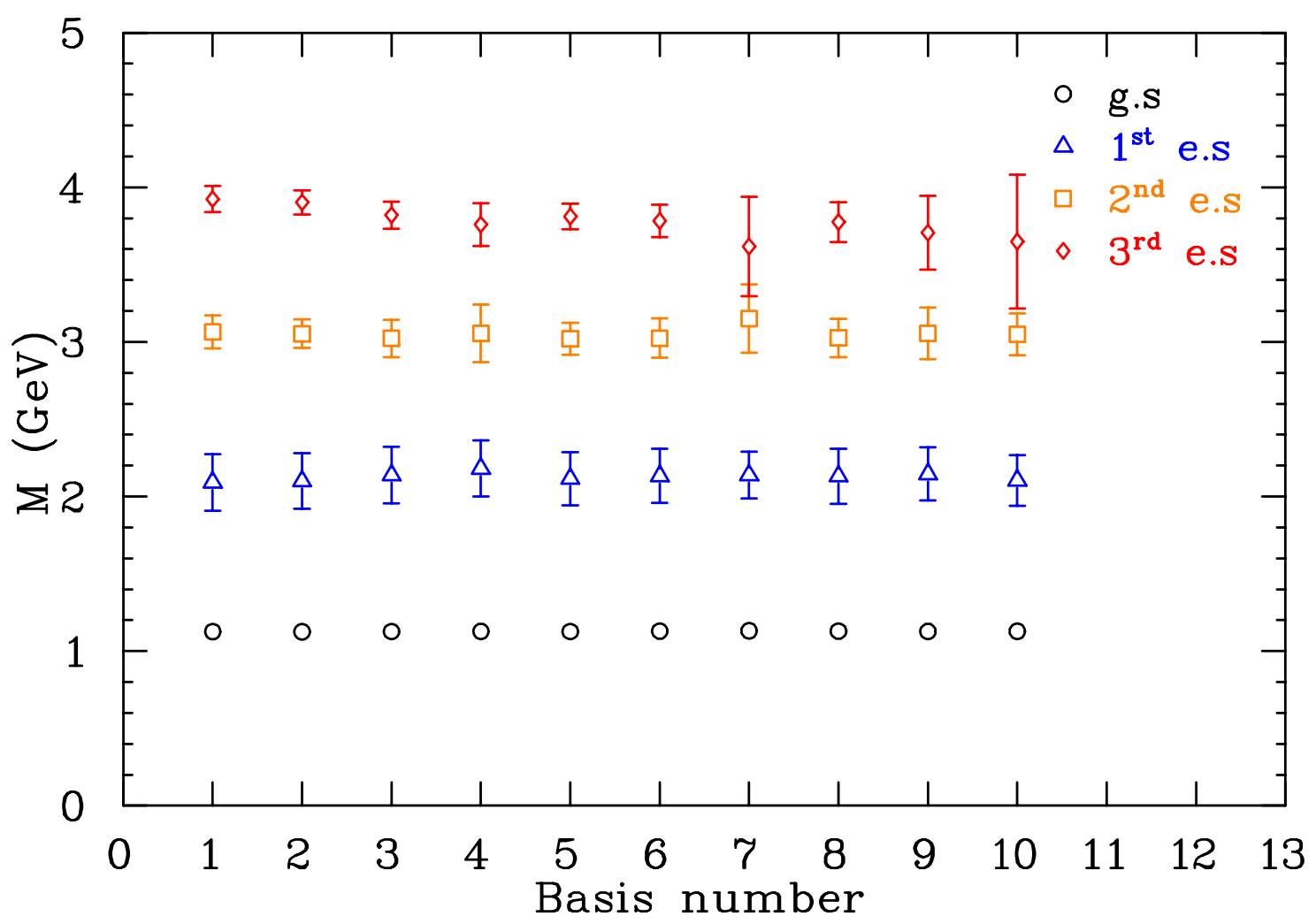

Figure 1: (Color online). Masses of the $\mathrm{N}_{2}{ }^{+}$energy states for various $4 \times 4$ correlation matrices (bases), given in Table 1, for $k_{u d}=0.13770$, over 50 configurations.

It is noted that basis operators that are linearly dependent will cause the eigenvalue analysis to fail as there will be a singularity in the correlation matrix. The fact that our analysis succeeds indicates that our choice of operators access an equal number of dimensions in the Hilbert space. It 
is interesting to examine the stability of the masses to different choices of bases to ascertain whether one has reliably isolated single eigenstates of QCD. The relevant issues are: (i) whether or not the operators are sufficiently far from collinear that numerical errors do not prevent diagonalisation of the correlation matrix and, (ii) whether or not the states of interest have significant overlap with the subspace spanned by our chosen sets of operators. Since our correlation matrix diagonalisation succeeded, except at large Euclidean times where statistical errors dominate, we conclude that our operators are sufficiently far from collinear.

Basis numbers 4, 7, 9 and 10 contain higher smearing-sweep counts of 400 and 800, which results in a significant enhancement of errors for the second and third excited states. It is noted that the sources with sweep counts of 400,800 and 1600 are very challenging as the smearing radii for these sources are close to the wall source. The poor signal-to-noise ratio for these sources make the correlation matrix analysis more challenging and the eigenvalue analysis becomes unsuccessful for a large number of variational parameters $\left(t_{0}, \triangle t\right)$. Therefore, the sources 400,800 and 1600 are undesirable to work with.

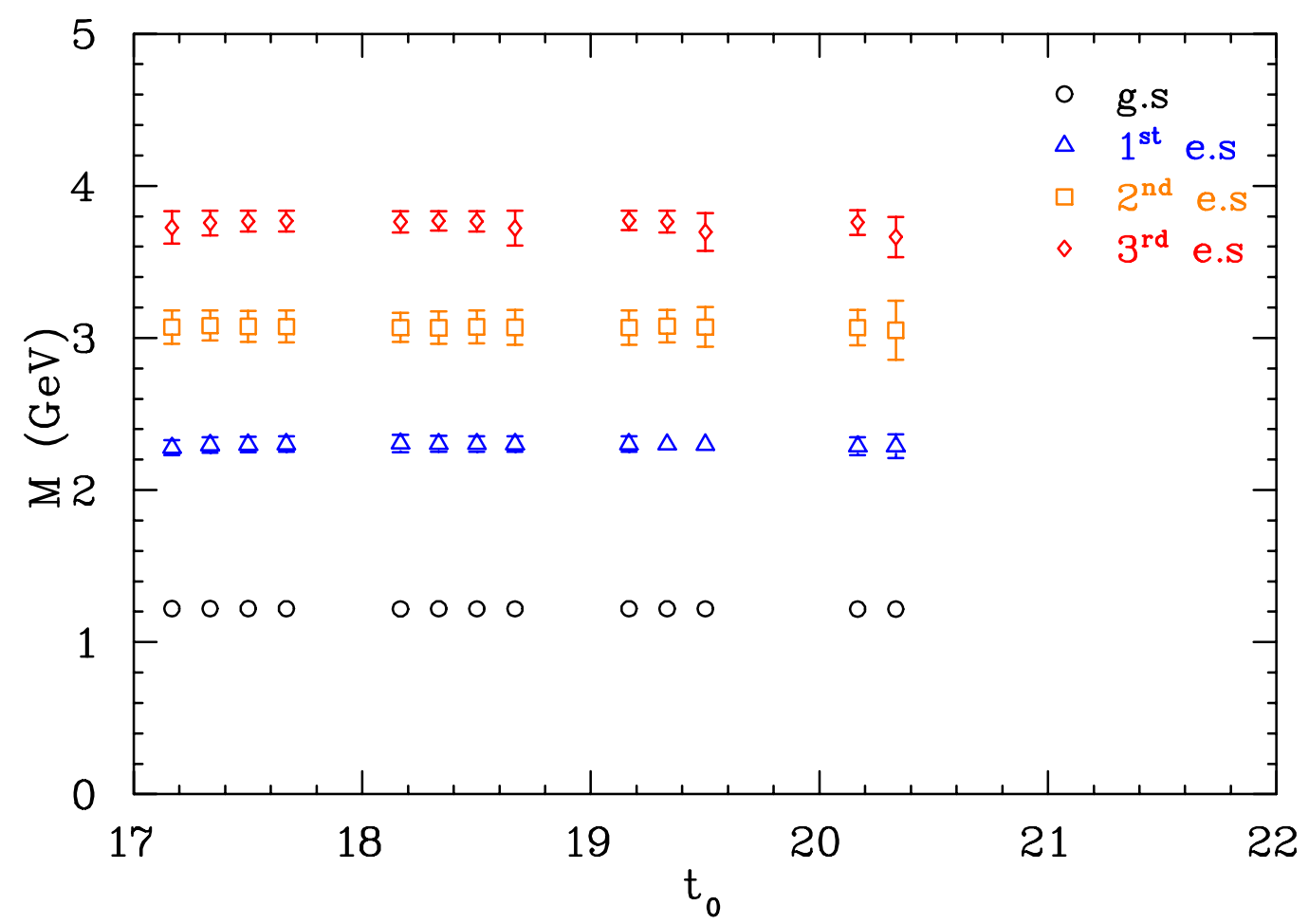

Figure 2: (Color online). Masses of the nucleon, $N \frac{1}{2}^{+}$states, from the projected correlation functions as shown in Eq. (2.7). Each set of ground (g.s) and excited (e.s) states masses correspond to the diagonalization of the correlation matrix for each set of variational parameters $t_{0}$ (shown in major tick marks) and $\triangle t$ (shown in minor tick marks). Figure corresponds to $k_{u d}=0.13754$ and for the 3 rd basis.

The agreement among the three lowest lying eigenstates is remarkable and verifies that our approach successfully isolates true eigenstates $[6,14]$.

Basis number 3 has good diversity including both lower and higher smearings which is necessary for the extraction of masses over the entire heavy to light quark mass range. As a result, basis 


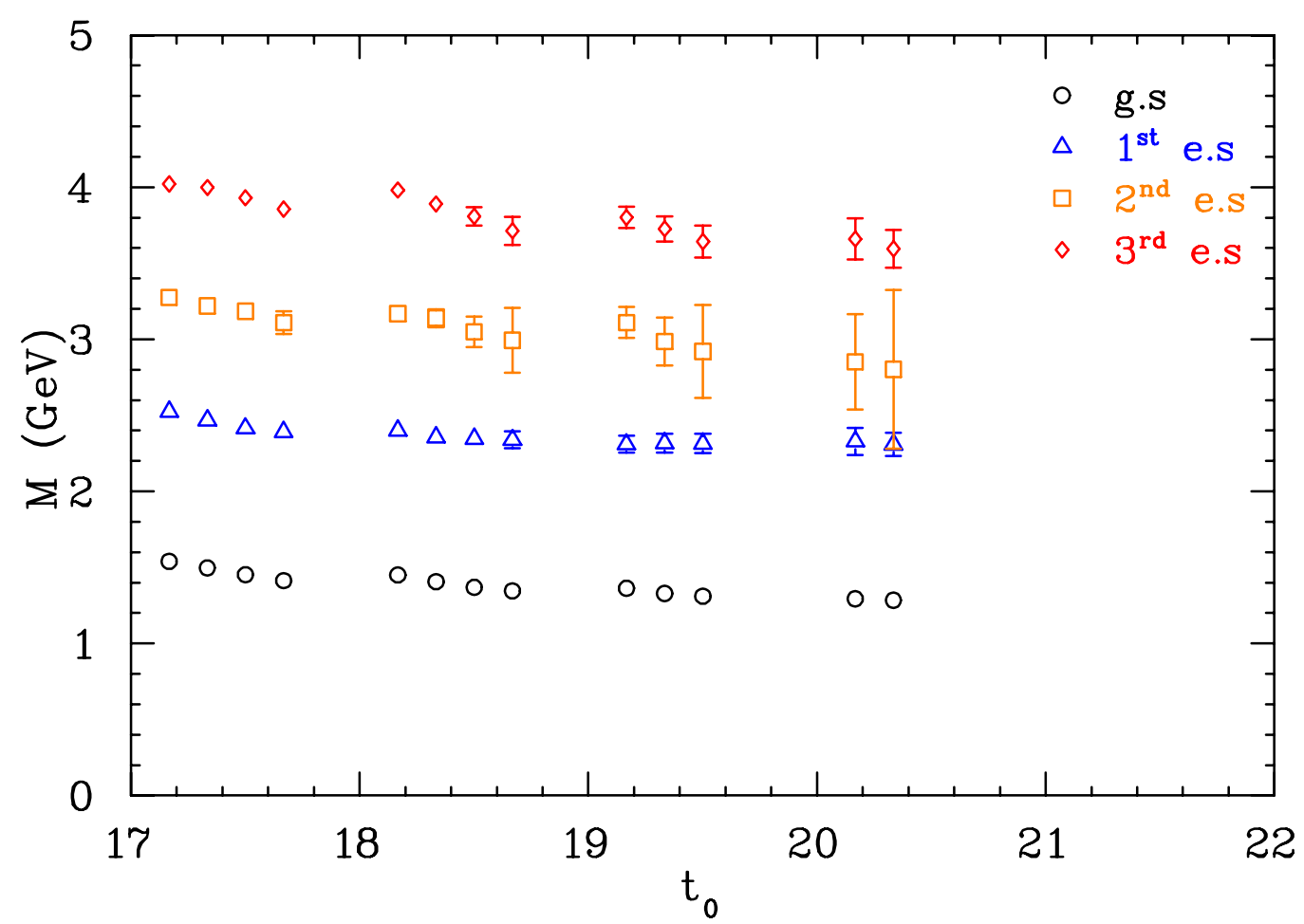

Figure 3: (Color online). As in Fig. 2, but masses are calculated from the eigenvalues as shown in Eqs. (2.4) and (2.5).

number 3 is considered for the following more extensive correlation-matrix analysis.

Consistency of the extracted masses from projected correlation functions over the variational parameters $t_{0}$ and $\triangle t$ is evident in Fig. 2, whereas a significant dependence of the extracted masses from the eigenvalues is evident in Fig. 3. Asymptotically, these energies agree with those of Fig. 2. These aspects of the extracted masses on variational parameters is in complete accord with our quenched analysis presented in Refs. [6, 7, 8, 14].

\section{Conclusions}

In these proceedings, we have presented a systematic correlation matrix analysis method using a variety of fermion field smearings at the source and sink to extract excited-state energies of the nucleon. Of particular interest is the Roper resonance from 2+1 flavor QCD. The negative parity channel will also be investigated to obtain information about the level orderings between the Roper and $N \frac{1}{2}^{-}$ground state in full QCD. Performing this analysis technique at all the quark masses will be the subject of future investigations.

\section{Acknowledgments}

We thank PACS-CS Collaboration for making these $2+1$ flavor configurations available and the ILDG [21] for creating the opportunity, tools and formalism for sharing these configurations. This 
research was undertaken on the NCI National Facility in Canberra, Australia, which is supported by the Australian Commonwealth Government. We also acknowledge eResearch SA for generous grants of supercomputing time which have enabled this project. This research is supported by the Australian Research Council.

\section{References}

[1] N. Isgur, G. Karl, Phys. Lett. B72 (1977) 109.

[2] N. Isgur, G. Karl, Phys. Rev. D19 (1979) 2653.

[3] D. B. Leinweber, Phys. Rev. D51 (1995) 6383-6393. [arXiv:nucl-th/9406001].

[4] C. Michael, Nucl. Phys. B259 (1985) 58.

[5] M. Luscher, U. Wolff, Nucl. Phys. B339 (1990) 222-252.

[6] M. S. Mahbub, et al., Phys. Lett. B679 (2009) 418-422. [arXiv:0906.5433].

[7] M. S. Mahbub, A. O. Cais, W. Kamleh, D. B. Leinweber, A. G. Williams, [arXiv:1004.5455].

[8] M. S. Mahbub, W. Kamleh, D. B. Leinweber, A. O. Cais, A. G. Williams, Phys. Lett. B693 (2010) 351-357. [arXiv:1007.4871].

[9] J. M. Bulava, et al., Phys. Rev. D79 (2009) 034505. [arXiv:0901.0027].

[10] J. Bulava, et al., Phys. Rev. D82 (2010) 014507. [arXiv:1004.5072].

[11] G. P. Engel, C. B. Lang, M. Limmer, D. Mohler, A. Schafer, Phys. Rev. D82 (2010) 034505. [arXiv:1005.1748].

[12] S. Aoki, et al., Phys. Rev. D 79 (2009) 034503. [arXiv:0807.1661].

[13] W. Melnitchouk, et al., Phys. Rev. D67 (2003) 114506. [arXiv:hep-lat/0202022].

[14] M. S. Mahbub, et al., Phys. Rev. D80 (2009) 054507. [arXiv:0905.3616].

[15] B. Blossier, M. Della Morte, G. von Hippel, T. Mendes, R. Sommer, JHEP 04 (2009) 094. [arXiv:0902.1265].

[16] Y. Iwasaki UTHEP-118.

[17] S. Aoki, et al., Phys. Rev. D73 (2006) 034501. [arXiv:hep-lat/0508031]

[18] R. Sommer, Nucl. Phys. B411 (1994) 839-854. [arXiv:hep-lat/9310022]

[19] S. Gusken, Nucl. Phys. Proc. Suppl. 17 (1990) 361-364.

[20] D. B. Leinweber, R. M. Woloshyn, T. Draper, Phys. Rev. D43 (1991) 1659-1678.

[21] http://ildg.sasr.edu.au/Plone/ildg/ 